\title{
MODELOS MATEMÁTICOS PARA A ESTIMATIVA DO POTENCIAL DE MINERALIZAÇÃO ANAERÓBIA DO NITROGÊNIO EM SOLOS DE VÁRZEA DO RIO GRANDE DO SUL ${ }^{(1)}$
}

\author{
Leandro Souza da Silva ${ }^{(2)}$, Anderson Clayton Rhoden ${ }^{(3)}$, Elisandra Pocojeski ${ }^{(4)}$, \\ Flávio Anastácio de Oliveira Camargo ${ }^{(5)}$ \& Eliziane Luiza Benedetti ${ }^{(6)}$
}

\section{RESUMO}

Diferentes modelos matemáticos são utilizados para estimar o potencial de mineralização de $\mathrm{N}$ no solo, e os parâmetros estimados com a decomposição anaeróbia podem ser empregados na predição da disponibilidade do $\mathrm{N}$ para a cultura do arroz irrigado. Os objetivos deste trabalho foram estimar, a partir de cinco modelos matemáticos, os parâmetros " $N$ potencialmente mineralizável" $\left(\mathrm{N}_{0}\right)$ e "taxa de mineralização" (k) de diferentes solos de várzea do Rio Grande do Sul (RS) e correlacionar os valores de $\mathrm{N}_{0}$ dos modelos com o $\mathrm{N}$ acumulado por plantas de arroz irrigado. O trabalho foi desenvolvido a partir de amostras de solos de várzea coletadas em 15 locais do RS, utilizadas em um experimento de incubação anaeróbia em laboratório, onde foram determinados valores de $\mathrm{N}$ mineral da solução do solo ao longo de 24 semanas. A partir dos resultados, foram estimados os parâmetros de acordo com cinco modelos matemáticos. Posteriormente, amostras dos solos foram utilizadas em dois cultivos sucessivos de arroz irrigado, em casa de vegetação, obtendo-se valores da quantidade de $\mathrm{N}$ acumulado pelas plantas. $\mathrm{O}$ modelo mais bem ajustado na estimativa do $\mathrm{N}_{0}$ e $\mathrm{k}$ para a média dos 15 solos de várzea do $\mathrm{RS}$ foi o de Jones. Entretanto, o $\mathrm{N}_{0}$ do modelo de Stanford \& Smith foi o que melhor se correlacionou com a quantidade de $\mathrm{N}$ acumulado pelas plantas de arroz irrigado no primeiro cultivo, enquanto o $\mathrm{N}_{0}$ do modelo de Jones foi o que melhor se correlacionou com o $\mathrm{N}$ acumulado pelas plantas no segundo cultivo.

Termos de indexação: arroz irrigado, disponibilidade de $\mathrm{N}$, decomposição anaeróbia.

\footnotetext{
(1) Parte da Tese de Mestrado do segundo autor apresentada a Universidade Federal de Santa Maria - UFSM. Recebido para publicação em outubro de 2007 e aprovado em maio de 2008

(2) Professor do Departamento de Solos, Centro de Ciências Rurais, Universidade Federal de Santa Maria - UFSM. Av. Roraima 1000, CEP 97105-900 Santa Maria (RS). E-mail: leandro@smail.ufsm.br

(3) Professor do curso de Agronomia da Faculdades de Itapiranga - FAI. Rua Carlos Kummer 100, Bairro Universitário, CEP 89896000 Itapiranga (SC). E-mail: andersonrhoden@mail.ufsm.br

(4) Doutoranda do Programa de Pós-Graduação em Ciência do Solo, Centro de Ciências Rurais, UFSM. E-mail: eliagro@mail.ufsm.br

(5) Professor do Departamento de Solos, Universidade Federal do Rio Grande do Sul - UFRGS. Av. Bento Gonçalves 7712, Caixa Postal 15100, CEP 91501-970 Porto Alegre (RS). E-mail: fcamargo@ufrgs.br

(6) Doutoranda do Programa de Pós-Graduação em Solos e Nutrição de Plantas, Universidade Federal de Viçosa - UFV. Av. P.H. Rolfs s/n. Campus Universitário, CEP 36570-000 Viçosa (MG). E-mail: elibettiagro@yahoo.com.br
} 


\title{
SUMMARY: MATHEMATICAL MODELS TO ESTIMATE THE ANAEROBIC NITROGEN MINERALIZATION POTENTIAL IN PADDY SOILS OF RIO GRANDE DO SUL, BRAZIL
}

\begin{abstract}
Mathematical models are used to estimate the nitrogen $(N)$ mineralization potential in the soil. The parameters of anaerobic decomposition can be used to predict $N$ availability to flooded rice. The objective of this study was to determine the "potentially mineralizable $N$ " $\left(N_{0}\right)$ and the "mineralization rate" $(\mathrm{k})$ parameters, using five mathematical models for different paddy soils of the state of Rio Grande do Sul (RS), Brazil, and to correlate the $N_{0}$ values from the models with $N$ uptake of flooded rice plants. The experiment was carried out with samples of paddy soils collected at 15 sites in $R S$, and used in one anaerobic incubation laboratory trial, where mineral $N$ values of the soil solution were determined during 24 weeks. The parameters were calculated based on mineral $N$ values using five mathematic models. The soil samples were used in two successive flooded rice crops, in a greenhouse, to obtain the quantity of plant $N$ uptake. The best model to estimate $N_{0}$ and $k$, averaged across the 15 paddy soils, was the Jones model. The parameter $N_{0}$ of the Stanford \& Smith model and $N_{0}$ of the Jones model correlated best with the $N$ uptake of flooded rice plants in the first and second crops, respectively.
\end{abstract}

Index terms: flooded rice, $N$ availability, anaerobic decomposition.

\section{INTRODUÇÃO}

O teor de matéria orgânica do solo (MOS) não é considerado um bom indicador da disponibilidade de $\mathrm{N}$ para o arroz irrigado, devido aos ciclos de oxidação e redução que os solos sofrem com o alagamento (Scivittaro \& Machado, 2004). Dessa forma, outras propriedades têm sido buscadas com o objetivo de avaliar adequadamente a disponibilidade de $\mathrm{N}$ às plantas ao longo do ciclo da cultura do arroz irrigado por alagamento. $\mathrm{O} \mathrm{N}$ potencialmente mineralizável $\left(\mathrm{N}_{0}\right)$ do solo e a taxa de mineralização do $\mathrm{N}(\mathrm{k})$, em condições anaeróbias, são índices que podem ser usados na predição da disponibilidade do $\mathrm{N}$ em determinado período de tempo, sendo o $\mathrm{N}_{0}$ definido como a fração do $\mathrm{N}$ orgânico do solo suscetível à mineralização e o $\mathrm{k}$ a taxa com que o N é mineralizado (Stanford \& Smith, 1972; Camargo et al., 1997). Os valores de $\mathrm{N}_{0}$ e $\mathrm{k}$, estimados por meio de resultados com experimentos de incubação-lixiviação em laboratório, poderiam ser utilizados como indicadores da disponibilidade de $\mathrm{N}$ às plantas, podendo-se ajustar à recomendação de adubação nitrogenada para o arroz irrigado em diferentes solos.

Entre os modelos empíricos que podem ser utilizados para obtenção desses índices (Camargo et al., 2002), alguns foram desenvolvidos para mineralização aeróbia e, ou, para solos oriundos de outras regiões, os quais podem não ser igualmente adequados para estimá-los em condições anaeróbias com solos de várzea, onde se cultiva arroz irrigado por alagamento no Rio Grande do Sul. O modelo matemático mais usado é o descrito por Stanford \& Smith (1972). Esses autores propuseram um modelo de cinética de primeira ordem, considerando somente um compartimento de $\mathrm{N}$ mineralizável, estimado em experimentos de incubação aeróbia de longa duração, que ocorre em função do tempo,em determinada taxa (Parentoni et al., 1988; Camargo et al., 1999). Comparando diversos modelos matemáticos para predição do $\mathrm{N}_{0}$ e k em 10 solos oxidados do RS, Camargo et al. (2002) observaram que os modelos exponenciais simples descrevem adequadamente a mineralização do N orgânico nos solos estudados.

Ao estudar a mineralização do $\mathrm{N}$ em seis solos chineses, Li et al. (2003) observaram que esta ocorreu em duas fases: a primeira, de 0-14 dias, considerada rápida, alcançou de 4 a $15 \mathrm{mg} \mathrm{kg} \mathrm{dia-1}$ de N no solo; e, a partir do $14^{\circ}$ dia, houve rápido declínio, fase considerada lenta. Pöttker \& Tedesco (1979), estudando a mineralização anaeróbia de N em solos do RS, comentaram que as taxas de mineralização desse nutriente diminuíram sensivelmente após as três primeiras semanas de incubação, concluindo que houve rápida liberação inicial de $\mathrm{N}$, seguida de diminuição gradativa. Já Camargo et al. (1997) observaram que essa diminuição ocorreu a partir da quarta semana de incubação, considerando que esse estudo foi realizado em condições aeróbias. Os resultados evidenciaram que a mineralização do $\mathrm{N}$ também poderia ser predita por modelos que assumem dois compartimentos, sugeridos como mais eficientes e que proporcionariam melhor adequação dos resultados do que os modelos de cinética de primeira ordem, considerando duas fases de $\mathrm{N}$ decomponíveis sob taxas diferentes (Lindemann \& Cardenas, 1984; Deans et al., 1986).

Jones propôs um modelo exponencial simples, que assume dois compartimentos de $\mathrm{N}$ potencialmente mineralizável, indicando que existe uma fração considerada fluxo inicial de $\mathrm{N}$ e outra fração ativa do 
N potencialmente mineralizável em determinada taxa (Jones, 1984). Camargo et al. (2002), no estudo dos modelos empíricos para predição da mineralização aeróbia do $\mathrm{N}$ em solos do RS, evidenciaram que o modelo proposto por Jones foi o que melhor descreveu o processo de mineralização e o $\mathrm{N}$ orgânico potencial para ser liberado ao ambiente e tornar-se disponível às plantas. Esses autores também afirmaram que este modelo foi o que melhor estimou o $\mathrm{N}$ no tempo zero, sendo essa variável matemática importante para a estimativa do $\mathrm{N}$ no início da mineralização.

O modelo matemático proposto por Inubushi et al. (1985) foi desenvolvido com o intuito de ajustar o N potencialmente mineralizável em solos alagados do Japão e das Filipinas, com incubação anaeróbia de 12 semanas. Com base em suas observações da variação do $\mathrm{N}$ mineral em função do tempo, esses autores propuseram dois compartimentos de mineralização, sendo um mais rápido (consumido no início do período de incubação) e outro mais lento (consumido posterior e continuamente até o final da incubação). Aplicando este modelo, Camargo et al. (2002) obtiveram resultados satisfatórios a partir da segunda semana de incubação aeróbia em 10 solos do RS.

O modelo parabólico de Broadbent (1986), que primeiramente foi descrito para determinar perdas de $\mathrm{C}$ de acordo com o tempo, também poderia estimar preliminarmente o $\mathrm{N}_{0}$ pelo ajuste não-linear (Camargo et al., 1999). Em um estudo que comparou o modelo proposto por Broadbent (1986) a outros sete modelos, Camargo et al. (2002) observaram que o primeiro modelo foi o que apresentou os resultados mais insatisfatórios na estimativa do $\mathrm{N}_{0}$ e do k. Cabrera (1993) comenta que, nos modelos que consideraram dois compartimentos de $\mathrm{N}$ mineralizável, um deles devia ser mineralizado de acordo com uma cinética de primeira ordem, e o outro, de acordo com uma cinética de ordem zero. Esse autor afirma que o modelo por ele proposto apresentou resultados significativamente melhores do que os modelos que consideram somente um compartimento de $\mathrm{N}$ mineralizável.

Os objetivos deste trabalho foram avaliar diferentes modelos matemáticos utilizados para estimativa do "N potencialmente mineralizável" e da "taxa de mineralização" em "N potencialmente mineralizável" e "taxa de mineralização" em diferentes solos de várzea do RS, a partir de dados de mineralização de $\mathrm{N}$ desses solos, e correlacionar com o $\mathrm{N}$ acumulado por plantas de arroz irrigado cultivadas em casa de vegetação.

\section{MATERIAL E MÉTODOS}

Foram utilizadas amostras de 15 solos de várzea, coletadas em 13 municípios do Estado do RS: Camaquã, Dom Pedrito, Uruguaiana (duas amostras), Caçapava do Sul, Santo Antônio da Patrulha, Santa Maria, São Gabriel, Cachoeirinha, Restinga Seca, Cachoeira do Sul (duas amostras), Santa Vitória do
Palmar, Rosário do Sul e Paraíso do Sul. A associação entre os locais de coleta dos solos e as unidades de mapeamento e a classificação brasileira foi realizada de acordo com Streck et al. (2002). Nas amostras, determinou-se o teor de $\mathrm{N}$ total e mineral, conforme Tedesco et al. (1995). O teor de argila foi determinado conforme método da Embrapa (1997), e o teor de C orgânico, conforme método de Nelson \& Sommers (1982) (Quadro 1). O pH $\mathrm{H}_{2 \mathrm{O}(1: 1 / \text { solo:água) }}$ o o índice SMP das amostras dos solos foram determinados segundo Tedesco et al. (1995) para que o $\mathrm{pH}$ pudesse ser corrigido para um valor próximo de 5,5 (CQFSRS/SC, 2004), padronizando-se assim os solos utilizados. Para a correção do $\mathrm{pH}$, foi utilizado calcário dolomítico comercial (PRNT $75 \%$ ). As amostras de solo com calcário foram colocadas dentro de sacos plásticos de $10 \mathrm{~kg}$ para incubação por 35 dias. Semanalmente abriram-se os sacos para que ocorressem as trocas gasosas.

Depois de incubadas, alíquotas de $4 \mathrm{~kg}$ de cada solo foram acondicionadas em vasos de $8 \mathrm{~L}$ para o cultivo, em casa de vegetação, de arroz irrigado, cultivar IRGA 417, em delineamento completamente casualizado e quatro repetições. Em cada solo, foram adicionados $\mathrm{P}$ na forma de $\mathrm{KH}_{2} \mathrm{PO}_{4}$, para atingir $0,2 \mathrm{mg} \mathrm{L}^{-1}$ de $\mathrm{P}$ na solução do solo, conforme a capacidade máxima de adsorção de $\mathrm{P}$ de cada um, e K na forma de $\mathrm{KCl}$ de modo a adicionar, em todos os solos, $82,65 \mathrm{mg} \mathrm{kg}^{-1}$ de $\mathrm{K}$, conforme procedimentos descritos em Ranno et al. (2007). Posteriormente, foram transplantadas oito plântulas de arroz, previamente germinadas, para cada vaso, e após cinco dias do transplante permaneceram seis plântulas por vaso. Aplicou-se uma lâmina de água de $5 \mathrm{~cm}$, com adição de água destilada quando necessário, até o final do experimento. A colheita da parte aérea das plantas de arroz irrigado foi realizada aos 60 dias após o transplante e secas em estufa de ventilação forçada a $60^{\circ} \mathrm{C}$. Posteriormente, as amostras foram pesadas, determinando-se a matéria seca produzida. Uma subamostra foi moída para determinação do teor de $\mathrm{N}$ total, conforme Tedesco et al. (1995). Com base na produção de matéria seca e no teor de $\mathrm{N}$ total do tecido das plantas, foi calculada a quantidade de $\mathrm{N}$ acumulado pelas plantas. Um segundo cultivo de arroz irrigado foi iniciado 11 dias após a colheita do primeiro cultivo, mantendo-se o solo alagado e realizando-se os mesmos procedimentos descritos anteriormente, exceto as adubações de $\mathrm{P}$ e $\mathrm{K}$.

Outra porção de cada solo, com o $\mathrm{pH}$ corrigido, foi utilizada, em laboratório, para o estudo de potencial de mineralização do N, com tubos de lixiviação $(100 \mathrm{~mL})$ em triplicatas. Cada tudo era composto por três camadas: superior, de $25 \mathrm{~g}$ de solo misturado à igual quantidade de areia lavada; intermediária, de $25 \mathrm{~g}$ de areia lavada; e inferior, de $2 \mathrm{~cm}$ de lã-de-vidro. Aos tubos foram adicionados $30 \mathrm{~mL}$ de solução contendo $\mathrm{MgSO}_{4}\left(0,002 \mathrm{~mol} \mathrm{~L}^{-1}\right)$ e $\mathrm{Ca}\left(\mathrm{H}_{2} \mathrm{PO}_{4}\right)_{2}$ $\left(0,005 \mathrm{~mol} \mathrm{~L}^{-1}\right)$, conforme Pottker \& Tedesco (1979). Os tubos foram cobertos com filme plástico, com um orifício central para trocas gasosas. Posteriormente, 
Quadro 1. Municípios de coleta das amostras dos solos, unidades de mapeamento, classificação brasileira e teores de carbono orgânico, nitrogênio total, argila e nitrogênio mineral dos solos

\begin{tabular}{|c|c|c|c|c|c|c|}
\hline Município & $\begin{array}{c}\text { Unidade de } \\
\text { mapeamento }^{(1)}\end{array}$ & $\begin{array}{l}\text { Classificação } \\
\text { brasileira }^{(1)}\end{array}$ & C orgânico & $\mathbf{N}$ total & Argila & $\begin{array}{c}\mathrm{N} \text { mineral } \\
\left(\mathrm{NH}_{4}^{+}+\mathrm{NO}_{3}^{-}\right)\end{array}$ \\
\hline & & & & $\mathrm{gg}^{-1}$ & & $\mathrm{mg} \mathrm{kg}^{-1}$ \\
\hline Camaquã & Itapeva & Gleissolo Melânico & 37,2 & 4,2 & 800 & 125,9 \\
\hline Dom Ped rito & Banhado & Gleissolo Háplico & 11,1 & 3,2 & 440 & 64,6 \\
\hline Uruguaiana 1 & Escobar & Vertissolo Ebânico & 30,9 & 3,9 & 370 & 139,0 \\
\hline Uruguaiana 2 & Uruguaiana & Chernossolo Ebânico & 20,0 & 2,6 & 330 & 98,2 \\
\hline Caçapava do Sul & São Gabriel & Planossolo Háplico & 16,2 & 2,0 & 320 & 52,5 \\
\hline Santo Antônio da Patrulha & Vacacaí & Planossolo Hidromórfico & 11,8 & 1,9 & 250 & 61,7 \\
\hline Santa Maria & Vacacaí & Planossolo Hidromórfico & 9,5 & 1,8 & 240 & 70,5 \\
\hline São Gabriel & São Gabriel & Planossolo Háplico & 13,8 & 1,8 & 230 & 105,5 \\
\hline Cachoeirinha & Banhado & Gleissolo Háplico & 10,9 & 1,7 & 200 & 76,3 \\
\hline Restinga Seca & Vacacaí & Planossolo Hidromórfico & 8,3 & 1,0 & 190 & 42,8 \\
\hline Cachoeira do Sul 1 & Vacacaí & Planossolo Hidromórfico & 5,6 & 1,1 & 180 & 39,9 \\
\hline Cachoeira do Sul 2 & Vacacaí & Planossolo Hidromórfico & 4,4 & 0,9 & 130 & 47,2 \\
\hline Santa Vitória do Palmar & Pelotas & Planossolo Hidromórfico & 9,2 & 1,3 & 120 & 90,9 \\
\hline Rosário do Sul & São Gabriel & Planossolo Háplico & 5,5 & 0,8 & 90 & 89,4 \\
\hline Paraíso do Sul & Vacacaí & Planossolo Hidromórfico & 7,3 & 0,7 & 80 & 51,5 \\
\hline
\end{tabular}

(1) Streck et al. (2002).

foram incubados em estufa a $30{ }^{\circ} \mathrm{C}$, sendo retirados somente para a coleta semanal da solução, até a $14^{\mathrm{a}}$ semana de incubação; a cada duas semanas, até a $18^{\mathrm{a}}$ semana; e a cada três semanas foram realizadas duas últimas coletas, totalizando 24 semanas de incubação anaeróbia (168 dias). A solução do solo foi drenada dos tubos com o auxílio de uma bomba de vácuo, sendo posteriormente pesada para que se adicionasse água destilada aos tubos num volume próximo aos $30 \mathrm{~mL}$ iniciais. O procedimento detalhado para isso encontra-se descrito em Rhoden et al. (2006). Na solução drenada dos tubos, foi determinado o teor de N mineral, conforme Tedesco et al. (1995).

Com os resultados estimados durante o período de incubação anaeróbia de 24 semanas, aplicaram-se cinco modelos matemáticos para o $\mathrm{N}$ mineralizável $\left(\mathrm{N}_{\mathrm{m}}\right)$, cuja expressão está descrita a seguir: (1) $\mathrm{N}_{\mathrm{m}}=$ $\mathrm{N}_{0}\left(1-\exp ^{-\mathrm{kt}}\right)$, proposto por Stanford \& Smith (1972), sendo $\mathrm{N}_{0}=\mathrm{N}$ potencialmente mineralizável; $\mathrm{k}=$ taxa de mineralização; (2) $\mathrm{N}_{\mathrm{m}}=\mathrm{N}_{1}+\mathrm{N}_{2}-\left(\mathrm{N}_{2} \exp ^{-\mathrm{k}}{ }_{2}^{\mathrm{t}}\right)$, proposto por Jones (1984), sendo $\mathrm{N}_{1}=\mathrm{N}$ mineral inicial; $\mathrm{N}_{2}=\mathrm{N}$ potencialmente mineralizável; $\mathrm{k}_{2}=$ taxa de mineralização; (3) $\mathrm{N}_{\mathrm{m}}=\mathrm{N}_{0 \mathrm{q}}(1$ - exp-kqt) $+\mathrm{N}_{0 \mathrm{~s}}\left(1-\exp ^{-\mathrm{kst}}\right)$, proposto por Inubushi et al. (1985), sendo $\mathrm{N}_{0 \mathrm{q}}=\mathrm{N}$ potencialmente mineralizável da fração lábil; $\mathrm{k}_{\mathrm{S}}=$ taxa de mineralização da fração lábil; $\mathrm{N}_{0 \mathrm{~s}}=\mathrm{N}$ potencialmente mineralizável da fração recalcitrante; $\mathrm{k}_{\mathrm{s} 2}=$ taxa de mineralização da fração recalcitrante; (4) $\mathrm{N}_{\mathrm{m}}=\mathrm{A} \mathrm{t}{ }^{\mathrm{b}}$, proposto por Broadbent (1986), sendo $\mathrm{A}=\mathrm{N}$ potencialmente mineralizável; $\mathrm{b}=$ taxa de mineralização; e (5) $\mathrm{N}_{\mathrm{m}}=\mathrm{N}_{1}\left(1-\exp ^{-\mathrm{k}}{ }_{1} \mathrm{t}\right)$ $+\mathrm{k}_{0} \mathrm{t}$, proposto por Cabrera (1993), sendo $\mathrm{N}_{1}=\mathrm{N}$ potencialmente mineralizável; $\mathrm{k}_{1}=$ taxa de mineralização; $\mathrm{k}_{0}=$ taxa de mineralização da fração recalcitrante.
As estimativas do potencial de mineralização do N e das taxas de mineralização e os parâmetros de ajuste dos modelos foram estimados com auxílio dos programas SAS-Proc Nlin, usando o algoritmo de Marquardt e SigmaPlot, a partir dos dados de N mineralizado acumulado em função do tempo de incubação anaeróbia. Também foram obtidas correlações lineares de Pearson $(p<0,01)$ entre os valores de $\mathrm{N}$ potencialmente mineralizável de cada modelo, com a quantidade de $\mathrm{N}$ absorvida pelas plantas de arroz irrigado em cada cultivo.

\section{RESULTADOS E DISCUSSÃO}

O modelo proposto por Stanford \& Smith apresentou elevado $\mathrm{R}^{2}(0,983)$, com $\mathrm{N}_{0}$ e $\mathrm{k}$ de $139,65 \mathrm{mg} \mathrm{kg}^{-1}$ e 0,2058 semana ${ }^{-1}$, respectivamente (Quadro 2). Esse é um modelo exponencial simples que considera somente um compartimento de $\mathrm{N}$ potencialmente mineralizável, o qual é decomposto a uma taxa proporcional ao tamanho deste compartimento (Broadbent, 1986; Camargo et al., 2002). Broadbent propôs um modelo parabólico que também considera somente um compartimento de $\mathrm{N}$ potencialmente mineralizável a uma taxa b (Broadbent, 1986). Esse modelo apresentou um $\mathrm{R}^{2} \mathrm{de}$ 0,88 , sendo o mais baixo entre os modelos avaliados. $\mathrm{O} \mathrm{N}$ potencialmente mineralizável estimado foi de 45,66 $\mathrm{mg} \mathrm{kg}^{-1}$, inferior ao observado nos demais modelos, e a taxa de mineralização foi de 0,3855 semana $^{-1}$, muito superior à dos outros, considerando a média dos 15 solos (Quadro 2). O modelo de Broadbent apresentou resultados inferiores da estimativa do potencial de mineralização do $\mathrm{N}$ e da taxa de 
mineralização em relação aos demais modelos. Também foi observado um desvio entre a curva estimada pelo modelo e a curva efetivamente obtida a partir dos valores medidos experimentalmente. Benbi \& Richter (2002) comentam que os valores de N mineralizado estimados pelos modelos de primeira ordem foram subestimados no início e no final do período de incubação e superestimados nos períodos intermediários. Entretanto, neste trabalho, observouse situação contrária para o modelo de Broadbent.

O modelo proposto por Inubushi e colaboradores, que conceitua dois compartimentos de $\mathrm{N}$ potencialmente mineralizável, cada um com uma taxa específica (Inubushi et al., 1985), apresentou um $\mathrm{R}^{2}=0,981$ na média dos 15 solos, sendo um pouco inferior ao estimado com o modelo de Stanford \& Smith. Na média dos 15 solos, a fração $\mathrm{N}_{0 \mathrm{q}}$, que é a fração de mineralização rápida de $\mathrm{N}$, foi de $80,53 \mathrm{mg} \mathrm{kg}^{-1}$, mineralizado a uma taxa de 0,2077 semana $^{-1}$. Já a fração $\mathrm{N}_{0 \mathrm{~s}}$, que é a fração de mineralização lenta do $\mathrm{N}$, foi de $59,06 \mathrm{mg} \mathrm{kg}^{-1}$, a uma taxa de 0,2077 semana-1 (Quadro 2). As taxas de mineralização do $\mathrm{N}$ apresentaram valores idênticos, não indicando a presença de dois compartimentos de $\mathrm{N}$ potencialmente mineralizável para os solos estudados, o que seria a principal vantagem desse modelo em relação ao anterior. Da mesma forma, quando se faz a soma do $\mathrm{N}$ potencialmente mineralizável estimado nos dois compartimentos propostos pelo modelo $(80,53+$ $59,06 \mathrm{mg} \mathrm{kg},-1, \mathrm{~N}_{0 \mathrm{q}}+\mathrm{N}_{0 \mathrm{~s}}$, respectivamente), obtémse um valor de $\mathrm{N}$ potencialmente mineralizável médio de $139,59 \mathrm{mg} \mathrm{kg}^{-1}$, a uma taxa de 0,2077 semana ${ }^{-1}$, sendo esse valor muito próximo dos já estimados pelo modelo de Stanford \& Smith. Essa pode ser a explicação para os modelos terem apresentado coeficientes de determinação muito próximos, sem vantagem do segundo modelo sobre o primeiro. Avaliando 32 semanas de incubação em ambiente aeróbio, Camargo et al. (2002) verificaram que não houve contribuição da fração recalcitrante para com o balanço do $\mathrm{N}$ orgânico mineralizado, corroborando as observações deste trabalho.

O modelo proposto por Cabrera também descreve dois compartimentos de $\mathrm{N}$ potencialmente mineralizável $\left(\mathrm{N}_{1}\right)$ (Cabrera, 1993), sendo o valor deste de $205,3 \mathrm{mg} \mathrm{kg}^{-1}$; o da taxa de mineralização do compartimento mais lábil $\left(\mathrm{k}_{1}\right), 0,1504$ semana $^{-1}$; e o da taxa de mineralização do $\mathrm{N}$ do compartimento mais estável $\left(\mathrm{k}_{0}\right)$ ou recalcitrante, - 2,7075 semana $^{-1}$, na média dos 15 solos estudados (Quadro 2). Este modelo apresentou elevado $R^{2}(0,983)$, idêntico ao estimado pelo modelo de Stanford \& Smith, mas com os menores valores para a soma de quadrados dos erros (SQe), quadrado médio do erro (QMe) e erro-padrão estimado (EPE), entre os modelos testados. Como o modelo considera dois compartimentos de $\mathrm{N}$ potencialmente mineralizável e o ajuste matemático do modelo se dá com base nesses dois compartimentos, o valor negativo da taxa de mineralização da fração recalcitrante também estaria indicando que não há um compartimento mais recalcitrante contribuindo com $\mathrm{N}$ mineralizado. $\mathrm{O}$ valor do $\mathrm{N}$ potencialmente mineralizável produzido por este modelo foi o maior entre os modelos avaliados, além das menores taxas de mineralização para o compartimento mais lábil, o que foi devido ao valor negativo estimado para o $\mathrm{k}_{0}$, considerando que os valores foram estimados pelo ajuste da equação. Este modelo, assim como o de Inubushi et al. (1985), permite sugerir que, para os 15 solos estudados em incubação anaeróbia durante um período de 24 semanas, não houve duas fases de mineralização do $\mathrm{N}$ e que a fração mais estável da MOS não foi decomposta ou apresentou pequena contribuição para com o $\mathrm{N}$ mineralizado nesse período.

Quadro 2. Média dos parâmetros dos modelos estimados pelo ajuste do N orgânico mineralizado acumulado em 24 semanas de incubação anaeróbia para 15 solos de várzea do RS

\begin{tabular}{|c|c|c|c|c|c|c|c|c|c|}
\hline Modelo & Fração do $\mathrm{N}_{0}$ & Fração k & $\mathbf{r}$ & $\mathbf{R}^{2}$ & $\mathbf{R}^{2} \mathbf{a j}$ & GLe & SQe & QMe & EPE \\
\hline $\begin{array}{l}\text { Stanford \& } \\
\text { Smith (1) }\end{array}$ & $\mathrm{N}_{0}=139,65$ & $\mathrm{k}=0,2058$ & 0,991 & 0,983 & 0,980 & 17 & 614,37 & 36,14 & 5,80 \\
\hline Inubushi et al.(2) & $\mathrm{N}_{0 \mathrm{~s}}=59,06$ & $\mathrm{k}_{\mathrm{s} 1}=0,2077 \quad \mathrm{k}_{\mathrm{s} 2}=0,2077$ & 0,990 & 0,981 & 0,977 & 15 & 614,37 & 40,96 & 6,18 \\
\hline Broadbent $^{(3)}$ & $\mathrm{A}=45,67$ & $\mathrm{~b}=0,3855$ & 0,938 & 0,880 & 0,880 & 17 & 3924,39 & 230,85 & 15,01 \\
\hline Cabrera $^{(4)}$ & $\mathrm{N}_{1}=205,29$ & $\mathrm{k}_{0}=-2,7070 \quad \mathrm{k}_{1}=0,1504$ & 0,992 & 0,983 & 0,980 & 16 & 393,02 & 24,56 & 5,07 \\
\hline Jones $^{(5)}$ & $\mathrm{N}_{2}=146,83$ & $\mathrm{~K}_{2}=0,2248$ & 0,996 & 0,991 & 0,990 & 16 & 471,97 & 29,50 & 5,11 \\
\hline
\end{tabular}

(1) $\mathrm{N}_{0}$ : N potencialmente mineralizável; $\mathrm{k}$ : taxa de mineralização. ${ }^{(2)} \mathrm{N}_{0 \mathrm{a}}: \mathrm{N}$ potencialmente mineralizável da fração lábil; $\mathrm{k}_{\mathrm{s}}$ : taxa de mineralização da fração lábil; $\mathrm{N}_{0 \mathrm{~s}}$ : $\mathrm{N}$ potencialmente mineralizável da fração recalcitrante; $\mathrm{k}_{\mathrm{s} 2}$ : taxa de mineralização da fração recalcitrante. ${ }^{(3)} \mathrm{A}$ : $\mathrm{N}$ potencialmente mineralizável; b: taxa de mineralização. ${ }^{(4)} \mathrm{N}_{1}: \mathrm{N}$ potencialmente mineralizável; $\mathrm{k}_{1}$ : taxa de mineralização; $\mathrm{k}_{0}$ : taxa de mineralização da fração recalcitrante. ${ }^{(5)} \mathrm{N}_{1}: \mathrm{N}$ mineral inicial; $\mathrm{N}_{2}: \mathrm{N}$ potencialmente mineralizável; $\mathrm{k}_{2}$ : taxa de mineralização; $r$ : coeficiente de correlação; $R^{2}$ : coeficiente de determinação; $R^{2}$ aj: coeficiente de determinação ajustado; GLe: graus de liberdade do erro; SQe: soma de quadrados do erro; QMe: quadrado médio do erro; EPE: erro-padrão estimado. 
O modelo proposto por Jones considera um fluxo inicial de mineralização do $\mathrm{N}\left(\mathrm{N}_{1}\right)$ e uma fração ativa de mineralização do $\mathrm{N}\left(\mathrm{N}_{2}\right)$ em função do tempo a uma taxa $\mathrm{k}_{2}$ (Camargo et al., 2002). O valor para o $\mathrm{N}_{1}$, na média dos 15 solos, foi de $-8,45 \mathrm{mg} \mathrm{kg}^{-1}$, o que também evidencia que não houve um fluxo inicial de mineralização do $\mathrm{N}$ para os solos estudados. Entretanto, Benbi \& Richter (2002) comentam que valores negativos poderiam indicar que está ocorrendo uma potencial imobilização do $\mathrm{N}$ do solo, que pode estar relacionada à decomposição da fração mais lábil da MOS e à incorporação do $\mathrm{N}$ na biomassa microbiana. A fração ativa $\mathrm{N}_{2}$ apresentou um valor de $146,8 \mathrm{mg} \mathrm{kg}^{-1}$ e a taxa de mineralização de 0,2248 semana $^{-1}$, na média dos 15 solos (Quadro 2). O R $\mathrm{R}^{2}$ foi o maior entre os modelos avaliados $(0,991)$, além de baixos valores para os parâmetros SQe, QMe e EPE, superiores apenas ao modelo proposto por Cabrera. Cabe salientar que a subtração do parâmetro $\mathrm{N}_{2}$ pelo parâmetro $\mathrm{N}_{1}$ produz resultados semelhantes com relação ao valor do $\mathrm{N}_{0}$ estimado pelo modelo de Stanford \& Smith, como já observado para o modelo de Inubushi e colaboradores.

Comparando os cinco modelos estudados na estimativa do $\mathrm{N}_{0}$ e do k para um período de incubação anaeróbia de 24 semanas, verifica-se que o modelo de Jones apresentou o maior valor para $\mathrm{R}^{2}(0,991)$. Os modelos de Cabrera e Stanford \& Smith apresentaram $\mathrm{R}^{2}$ de 0,983 ; o modelo de Inubushi e colaboradores, de 0,981 ; e o menor valor para o $R^{2}$ foi estimado com o modelo de Broadbent de 0,88 , na média dos 15 solos (Quadro 2). Esses resultados evidenciam que os modelos exponenciais simples foram os mais eficientes em estimar o $\mathrm{N}_{0}$ e o k; desses, o modelo de Jones foi o que melhor estimou os parâmetros $\mathrm{N}$ potencialmente mineralizável e taxa de mineralização (Quadro 3). Camargo et al. (1999) comentam que os modelos exponenciais duplos não estimam adequadamente frações do $\mathrm{N}$ do solo. Camargo et al. (2002), ao avaliarem modelos empíricos para predizer o $\mathrm{N}_{0}$ e o $\mathrm{k}$, observaram que eles não melhoraram a estimativa do $\mathrm{N}_{0}$ e do $\mathrm{k}$ em relação aos exponenciais simples, além de que os modelos com mais de três parâmetros não produziram os melhores resultados devido ao baixo número de graus de liberdade, corroborando os resultados observados.

A quantidade de $\mathrm{N}$ acumulado pelas plantas de arroz irrigado durante um período de 60 dias, de dois cultivos sucessivos, variou de 24,7 a $307,7 \mathrm{mg} / \mathrm{vaso}$ no primeiro e 9,84 a $113,59 \mathrm{mg} /$ vaso no segundo cultivo, o que demonstra que existem grandes diferenças entre os solos de várzea do RS estudados quanto à capacidade de mineralização e disponibilidade do N. A maior correlação entre o $\mathrm{N}_{0}$ estimado e a quantidade de $\mathrm{N}$ acumulado pelas plantas em cada solo, no primeiro cultivo de arroz em casa de vegetação, foi obtida com o modelo de Stanford \& Smith $(r=0,93$, $\mathrm{p}<0,01$ ) (Figura 1a). A correlação entre a quantidade de $\mathrm{N}$ acumulado pelas plantas no segundo cultivo de arroz irrigado e o $\mathrm{N}_{0}$ também foi significativa $(\mathrm{r}=$ $0,70, p<0,01$ ), apesar de o coeficiente de correlação não ter sido o maior entre os modelos. Cabe salientar que, quando se exclui o solo de Camaquã, a correlação cai para $0,46(p<0,05)$, demonstrando o comportamento diferenciado do segundo cultivo em relação ao primeiro, o que pode estar relacionado com a influência da contribuição do $\mathrm{N}$ mineralizado aerobiamente antes do primeiro cultivo (Quadro 1), o que não existiu quando da realização do segundo

Quadro 3. Parâmetros do modelo de Jones $\left[N_{m}=N_{1}+N_{2}-\left(N_{2} \exp ^{-k_{2}}{ }^{t}\right)\right]$ estimados pelo ajuste do $N$ orgânico mineralizado acumulado em 24 semanas de incubação anaeróbia de 15 solos de várzea do RS

\begin{tabular}{|c|c|c|c|c|c|c|c|c|c|}
\hline Solo & $\mathbf{N}_{1}$ & $\mathbf{N}_{2}$ & $\mathbf{K}_{2}$ & $\mathbf{r}$ & $\mathbf{R}^{2}$ & $\mathbf{R}^{2} \mathbf{a j}$ & SQe & QMe & EPE \\
\hline Camaquã & $-14,47$ & 218,28 & 0,1667 & 0,995 & 0,989 & 0,988 & 962,18 & 60,14 & 5,85 \\
\hline Dom Pedrito & $-4,16$ & 120,82 & 0,2136 & 0,994 & 0,989 & 0,987 & 190,14 & 11,88 & 4,05 \\
\hline Uruguaiana 1 & $-0,59$ & 183,98 & 0,1993 & 0,995 & 0,989 & 0,988 & 326,59 & 20,41 & 4,17 \\
\hline Uruguaiana 2 & $-9,89$ & 189,65 & 0,1982 & 0,999 & 0,998 & 0,996 & 546,95 & 34,18 & 4,20 \\
\hline Caçapava & $-12,84$ & 171,87 & 0,2086 & 0,994 & 0,989 & 0,988 & 893,11 & 55,82 & 6,08 \\
\hline Santo Antônio da Patrulha & $-10,16$ & 156,22 & 0,2118 & 0,995 & 0,989 & 0,988 & 603,43 & 37,71 & 5,42 \\
\hline Santa Maria & $-6,12$ & 143,82 & 0,2511 & 0,997 & 0,993 & 0,992 & 300,42 & 18,78 & 5,22 \\
\hline São Gabriel & $-5,63$ & 137,51 & 0,2417 & 0,990 & 0,981 & 0,979 & 240,44 & 15,03 & 4,25 \\
\hline Cachoeirinha & $-10,31$ & 139,45 & 0,2337 & 0,995 & 0,991 & 0,989 & 504,23 & 31,51 & 5,19 \\
\hline Restinga Seca & $-6,74$ & 107,67 & 0,2505 & 0,999 & 0,997 & 0,996 & 210,42 & 13,15 & 5,12 \\
\hline Cachoeira 1 & $-9,94$ & 125,95 & 0,2327 & 0,998 & 0,996 & 0,995 & 450,95 & 28,18 & 4,66 \\
\hline Cachoeira 2 & $-8,63$ & 128,12 & 0,2328 & 0,998 & 0,997 & 0,995 & 411,78 & 25,74 & 5,81 \\
\hline Santa Vitória do Palmar & $-5,66$ & 142,12 & 0,2563 & 0,995 & 0,991 & 0,990 & 259,26 & 16,20 & 5,25 \\
\hline Rosário do Sul & $-11,13$ & 122,24 & 0,2358 & 0,984 & 0,989 & 0,988 & 626,22 & 39,14 & 4,98 \\
\hline Paraíso do Sul & $-10,40$ & 114,65 & 0,2394 & 0,984 & 0,989 & 0,988 & 553,41 & 34,59 & 6,35 \\
\hline
\end{tabular}

$\overline{\mathrm{N}_{1}}$ : N mineral inicial; $\mathrm{N}_{2}: \mathrm{N}$ potencialmente mineralizável; $\mathrm{k}_{2}$ : taxa de mineralização; $\mathrm{r}$ : coeficiente de correlação; $\mathrm{R}^{2}$ : coeficiente de determinação; $\mathrm{R}^{2}$ aj: coeficiente de determinação ajustado; SQe: soma de quadrados do erro; QMe: quadrado médio do erro; EPE: erro-padrão estimado. 
cultivo, tendo em vista que os solos já estavam alagados. A fração ativa do $\mathrm{N}$ mineralizado $\left(\mathrm{N}_{2}\right)$ do modelo de Jones, para cada solo (Quadro 3), também apresentou correlação significativa com a quantidade de $\mathrm{N}$ acumulado pelas plantas no primeiro $(0,90$; $\mathrm{p}<0,01)$ e segundo cultivo $(0,71 ; \mathrm{p}<0,01)$, com valores de coeficiente de correlação semelhantes aos estimados pelo modelo de Stanford \& Smith, sendo um pouco menor no primeiro e um pouco maior no segundo cultivo (Figura 1b). Entretanto, quando se exclui o solo de Camaquã, a correlação cai para 0,43 $(\mathrm{p}<0,05)$, demonstrando o comportamento diferenciado do segundo cultivo em relação ao primeiro. Avaliando a correlação dos parâmetros de mineralização do $\mathrm{N}$ e a absorção de $\mathrm{N}$ pelo milho em 10 solos do RS, Camargo et al. (1999) observaram que o parâmetro $\mathrm{N}_{2}$ descrito por Jones foi o que melhor se correlacionou com a absorção de $\mathrm{N}$ pelo milho. Os resultados obtidos neste trabalho indicam que a estimativa do $\mathrm{N}_{0}$ pode ser usada como uma ferramenta importante na predição da disponibilidade de $\mathrm{N}$ às plantas de arroz irrigado por alagamento.

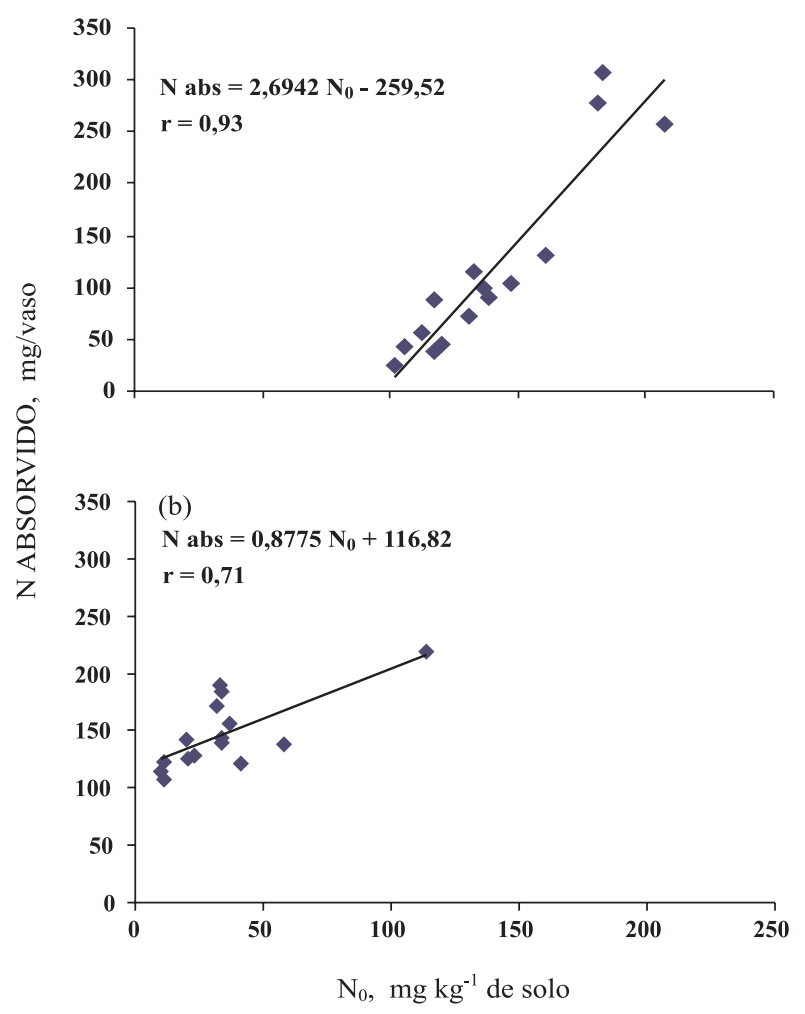

Figura 1. Correlação entre o teor de $\mathrm{N}$ acumulado por plantas de arroz irrigado no primeiro cultivo e o $\mathrm{N}$ potencialmente mineralizável $\left(\mathrm{N}_{0}\right)$, estimado pelo modelo de Stanford \& Smith (a), e o teor de $\mathrm{N}$ acumulado por plantas de arroz irrigado no segundo cultivo e a fração ativa do $\mathrm{N}$ potencialmente mineralizável, estimado pelo modelo de Jones (b), para 15 solos de várzea do RS.

\section{CONCLUSÕES}

1. Os modelos exponenciais simples foram os mais eficientes em estimar o $\mathrm{N}$ potencialmente mineralizável $\left(\mathrm{N}_{0}\right)$ e a taxa de mineralização $(\mathrm{k}) \mathrm{em}$ diferentes solos de várzea do RS, sendo o modelo de Jones o que melhor estimou os parâmetros $\mathrm{N}_{0}$ e k em 15 solos de várzea do RS, considerando o ajuste com o experimento de mineralização anaeróbia.

2. $\mathrm{O} \mathrm{N}_{0}$ do modelo de Stanford \& Smith foi o que melhor se correlacionou com a quantidade de $\mathrm{N}$ acumulado por plantas de arroz irrigado no primeiro cultivo, enquanto o $\mathrm{N}_{0}$ do modelo de Jones foi o que melhor se correlacionou com a quantidade de $\mathrm{N}$ acumulado pelas plantas no segundo cultivo, tendo os dois modelos apresentado um comportamento semelhante em ambos os cultivos.

\section{AGRADECIMENTOS}

Os autores agradecem a contribuição dos professores José Henrique Souza da Silva e Dalvan José Reinert, da UFSM, em relação aos tratamentos estatísticos e matemáticos dos dados deste trabalho.

\section{LITERATURA CITADA}

BENBI, D. \& RICHTER, J. A critical review of some approaches to modelling nitrogen mineralization. Biol. Fert. Soils, 35:168-183, 2002.

BROADBENT, F.E. Empirical modelling of soil nitrogen mineralization. Soil Sci., 141:208-213, 1986.

CABRERA, M.L. Modelling the flush of nitrogen mineralization caused by drying and rewetting soils. Soil Sci. Soc. Am. J., 57:63-66, 1993.

CAMARGO, F.A.O.; GIANELLO, C. \& VIDOR, C. Potencial de mineralização do nitrogênio em solos do Rio Grande do Sul. R. Bras. Ci. Solo, 21:575-579, 1997.

CAMARGO, F.A.O.; GIANELO, C. \& TEDESCO, M.J. Nitrogênio orgânico do solo. In: CAMARGO, F.A.O. \& SANTOS, F.A., eds. Fundamentos da matéria orgânica do solo: ecossistemas tropicais e subtropicais. Porto Alegre, Gênesis, 1999. p.117-137.

CAMARGO, F.A.O.; GIANELLO, C.; TEDESCO, M.J.; RIBOLDI, J.; MEURER, E.J. \& BISSANI, C.A. Empirical models to predict soil nitrogen mineralization. Ci. Rural, 12:393-399, 2002

COMISSÃO DE QUÍMICA E FERTILIDADE DO SOLO CQFSRS/SC. Manual de adubação e de calagem para os Estados do Rio Grande do Sul e Santa Catarina. 10.ed. Porto Alegre, 2004. 400p.

DEANS Jr.; MOLINA, J.A. \& CLAPP, C.E. Models for predicting potentially mineralizable nitrogen and decomposition rate constants. Soil Sci. Soc. Am. J., 50:323-326, 1986. 
EMPRESA BRASILEIRA DE PESQUSA AGROPECUÁRIA EMBRAPA. Centro Nacional de Pesquisa de Solos. Manual de métodos de análise de solo. Brasília, 1997. 212 p.

INUBUSHI, K.; WADA, H. \& TAKAI, Y. Easily decomposable organic matter in paddy soil. VI. Kinetics of nitrogen mineralization in submerged soils. Soil Sci. Plant Nutr., 4:563-572, 1985.

JONES, A. Estimation of an active fraction of soil nitrogen. Comm. Soil Sci. Plant Anal., 15:23-32, 1984.

LI, H.; HAN, Y. \& CAI, Z. Nitrogen mineralization in paddy soils of the Taihu region of China under anaerobic conditions: Dynamic and model fitting. Geoderma, 115:161-175, 2003.

LINDEMANN, W.C. \& CARDENAS, M. Nitrogen mineralization potential and nitrogen transformations of sludge-amended soils. Soil Sci. Soc. Am. J., 48:1072-1077, 1984.

NELSON, D.W. \& SOMMERS, L.E. Total carbon, organic carbon, and organic matter. In: PAGE, A. L., ed. Methods of soil analysis. 2.ed. Madison, American Society of Agronomy, 1982. Part 2. p.539-579.

PARENTONI, S.N.; FRANÇA, G.E. \& BAHIA FILHO, A.F.C. Avaliação dos conceitos de quantidade e intensidade de mineralização de nitrogênio para trinta solos do Rio Grande do Sul. R. Bras. Ci. Solo, 12:225-229, 1988.
PÖTTKER, D. \& TEDESCO, M.J. Efeito do tipo e tempo de incubação sobre a mineralização da matéria orgânica e nitrogênio total em solos do Rio Grande do Sul. R. Bras. Ci. Solo, 3:20-24, 1979.

RANNO, S.K.;. DA SILVA, L.S.; GATIBONI, L.C. \& RHODEN, A.C. Capacidade de adsorção de fósforo em solos de várzea do Estado do Rio Grande do Sul. R. Bras. Ci. Solo, 31:21$28,2007$.

RHODEN, A.C.; DA SILVA, L.S.; CAMARGO, F.A.O.; BRITZKE, D. \& BENEDETTI, E.L. Mineralização anaeróbia do nitrogênio em solos de várzea do Rio Grande do Sul. Ci. Rural. 36:1780-1787, 2006.

SCIVITTARO, W.B. \& MACHADO, M.O. Adubação e calagem para a cultura do arroz irrigado. In: GOMES, A.S. \& MAGALHÃES JUNIOR, A.M., orgs. Arroz Irrigado no Sul do Brasil. Brasília, Embrapa Informação Tecnológica, 2004. p.259-303.

STANFORD, G. \& SMITH, S.J. Nitrogen mineralization potential of soils. Soil Sci. Soc. Am. Proc., 36:465-472, 1972.

STRECK, E.V.; KÄMPF, N.; DALMOLIN, R.S.D.; KLAMPT, E.; NASCIMENTO, P.C. \& SCHINEIDER, P. Solos do Rio Grande do Sul. Porto Alegre, Universidade Federal do Rio Grande do Sul, 2002. 107p.

TEDESCO, M.J.; GIANELLO, C.; BISSANI, C.A.; BOHNEN, H. \& WOLKWEISS, S.J. Análises de solo, planta e outros materiais. 2.ed. Porto Alegre, Universidade Federal do Rio Grande do Sul, 1995. 174p. (Boletim Técnico, 5) 